\title{
METHOD TO RESOLVE DATA TRAFFIC ISSUES IN TELECOMMUNICATION SERVICE PROVIDERS NETWORKS
}

This paper was downloaded from TechRxiv (https://www.techrxiv.org).

\section{LICENSE}

CC BY-NC-SA 4.0

SUBMISSION DATE / POSTED DATE

19-01-2022 / 24-01-2022

\section{CITATION}

Narayanan, Priya; Seshadri, Veera Raghavan (2022): METHOD TO RESOLVE DATA TRAFFIC ISSUES IN TELECOMMUNICATION SERVICE PROVIDERS NETWORKS. TechRxiv. Preprint. https://doi.org/10.36227/techrxiv.18666470.v1

$\mathrm{DOI}$ 


\title{
METHOD TO RESOLVE DATA TRAFFIC ISSUES IN TELECOMMUNICATION SERVICE PROVIDERS NETWORKS
}

\author{
Veera Seshadri \\ Technology Lead, Cloud \\ Global Delivery Centre, India
}

\author{
Priya Narayanan \\ Technology Lead, Core \\ Global Delivery Centre, India
}

\begin{abstract}
Data Networks are constantly evolving from the times when voice was circuit switched until data services being delivered on packet switched network. Data networks are now hosting traditional voice services, incumbent data services and paving way to services which is time and data sensitive. It won't be long where almost everything around us will be connected to each other. The connectivity of the underlying data network plays a major role to ensure seamless services running on the top of this layer. Network Operations will undergo a remarkable transformation and reform the way they traditionally work. One of them would be to ensure proactive anomaly detection/ resolution, much faster and error free than how we were traditionally engineered. This paper discusses the most common use case, which is data network issues, that may be triggered due to an array of reasons. Often it is difficult to diagnose such cases in time unless we have the right tool or expertise. The paper highlights areas captured across most domains and puts it together for ease in understanding and implementation.
\end{abstract}

Index terms: SIP protocol, DIAMETER protocol, Analytics, EPC, IMS networks

\section{Surge in data Traffic and the challenges} towards end user experience

Telecom operators face unprecedented challenges owing to the exponential growth of data traffic. They are now in the crosshairs of conflicting trends - the rise of mobile network traffic and the decline of mobile subscriber revenue. Today, mobile traffic growth is driven mainly by Machine-to-Machine (M2M) interactions and video consumption.

In a landscape characterized by constant digital innovation, it is no longer enough to just effectively manage network functions.

Proactive network monitoring with increased technology deep-dive and approach towards boarder data related problems is mandatory not only to efficiently manage and operate the networks but also to gain customer's confidence.

\section{Problems faced with Slow Data browsing}

Few to list on the problems related to burst of data growth are the following:

- Severely impacting user experience which directly contributes to revenue loss to the operator, owing to customer leaving the network

- Considering the size and complexity of the today's network, lack of expertise contributing to increased CAPEX for the operator. Reason being quicker service restoration pressure not allowing to address the root cause of the problem and opting for Hardware replacement or just solving the issue

- Lack of standard operating procedure and missing E2E view on data connecting points

- Missing understanding of Proactive events and its corresponding correlation with reported alarms

\section{High level Architecture}

Figure-1 depicts the high-level architecture on different network element's interconnection involved during the data browsing traffic function in an LTE network. The values are standard and might vary depending on coverage and implementation.

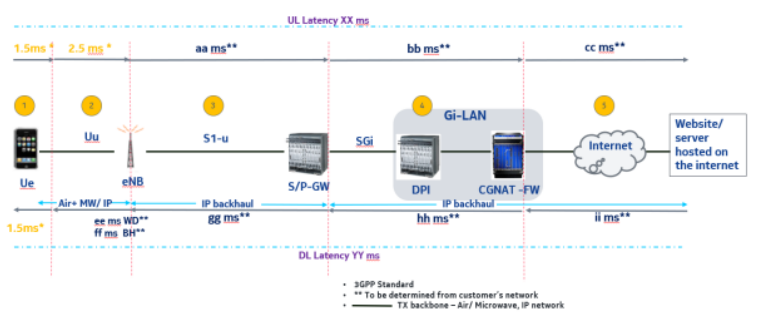

Figure 1: High Level Architecture

The latency values between any two nodes will be operator specific and to be available with the Operations team before debugging any related issues.

This is briefed below:

(a) Uu (UE --- eNB) latency value. Ideally it should be between 2.5 to $5 \mathrm{msec}$.

(b) UL latency. This will be sum of S1-U (eNB --- S/P GW) + other Application server in core network + SGi (P-GW --- GiLAN) interfaces.

(c) DNS resolution time

(d) step b + step c will be approximately between 50$75 \mathrm{msec}$

In this whitepaper, below listed functions are discussed on how it will impact the data services related problems and along with it the pro-active monitoring techniques to alleviate the problems.

- Throughput issues 
- Uplink/Downlink latency issues

- Intermittent slow data browsing

- Differential and OTT services issues

- GiLAN configuration issues

IV. Flow Chart to analyze slow data browsing issues The team can refer the flow chart to analyze data browsing related problems as depicted in Figure 2

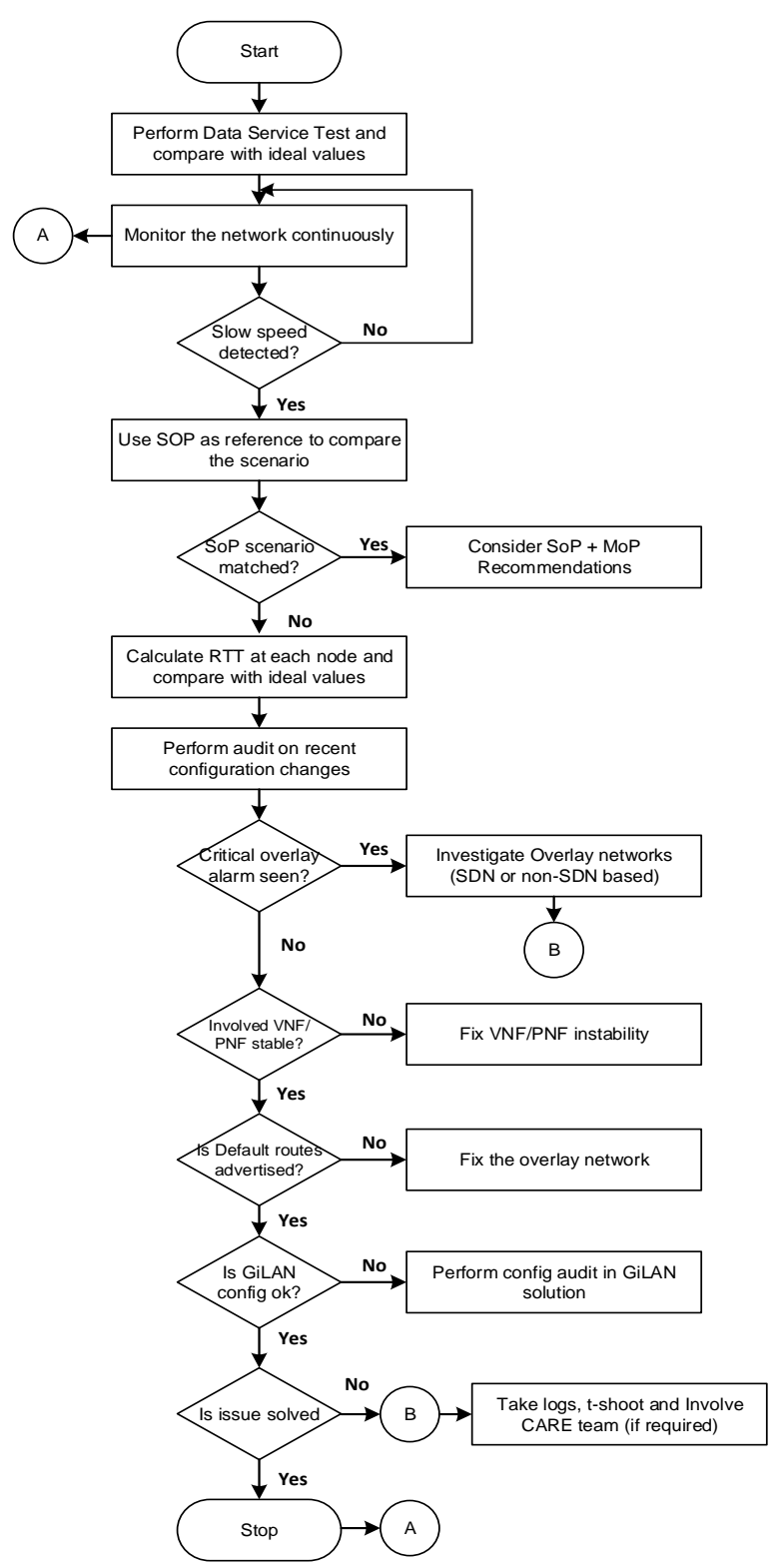

Figure 2: Flow chart on approach towards data browsing issues

\section{Pre-checks}

It is always better to have checklist ready and process defined when approaching data issues. Based on the analysis derived from a project incident, up to $60 \%$ of the issues are one of these types (human error, config error and process error). This section explains the various checks, an network engineer should perform as initial analysis.

\section{a. Subscriber profile}

Data provisioning for a subscriber must be verified both in HLR and Evolved Packet System (EPS) profiles.

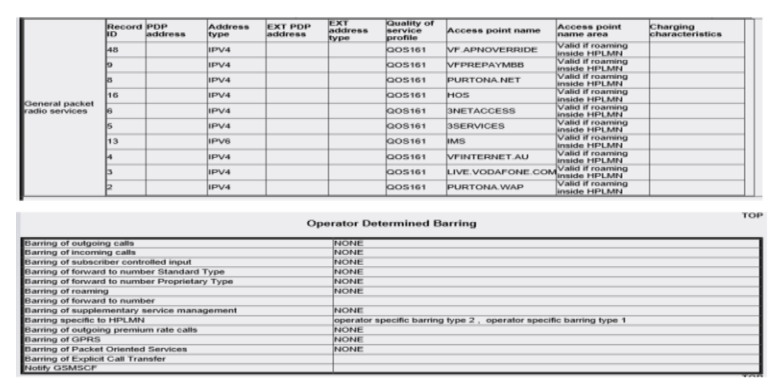

Figure 3: Sample HLR Profile check

For slow data browsing HLR profile and EPS profiles can be checked.

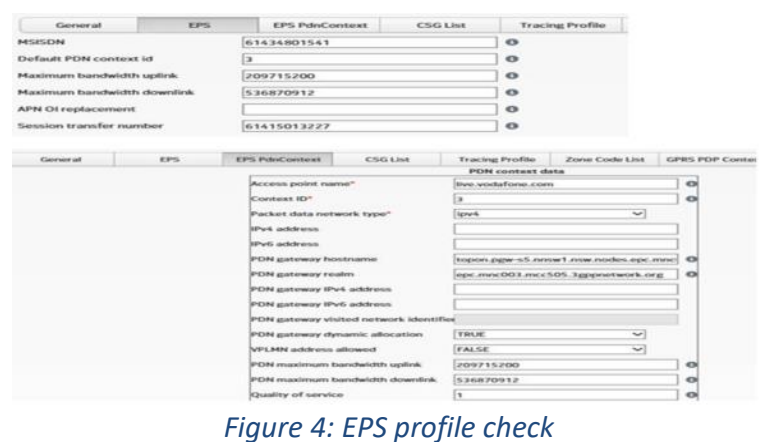

The below can be checked in these profiles:

- No data barring is set in subscriber profiles.

- Correct APN configured with maximum UL/DL bandwidth allocation per subscriber

- $\quad$ Check QoS profile (Operator specific)

- Check maximum UL/DL bandwidth configured for default PDN context in EPS profile

Note: For network where S4 interface being used, 3G subscriber will use the subscription profile of EPS profile which requires to be verified

\section{b. L2/L3 Network Pre-check}

Network level checks involves verifying:

- Packet loss

- Latency

- Cyclic Redundancy Check (CRC) error, this will occur in case of physical layer failures or malfunctioning

- BFD alarms (link failures)

- BGP alarms for neighborship or maximum threshold limit failures 


\section{Use cases and Scenario's}

This section explains the different possible issues that can occur in the network.

\section{a. TCP Proxy/MTU function contributing to slow browsing issue}

Timestamp Value field (TSVal) and MTU are the two important parameters to check in the uplink/downlink packets. TSVal denotes the timestamp value and if fluctuates will result in performance degradation. Figure 5 explains the anomaly observed with the packets originating from UE. TCP Proxy should not be sending a TSVal that jumps up and then back again, it is supposed to be monotonically non-decreasing. As a result, out of ordering being introduced in the network.

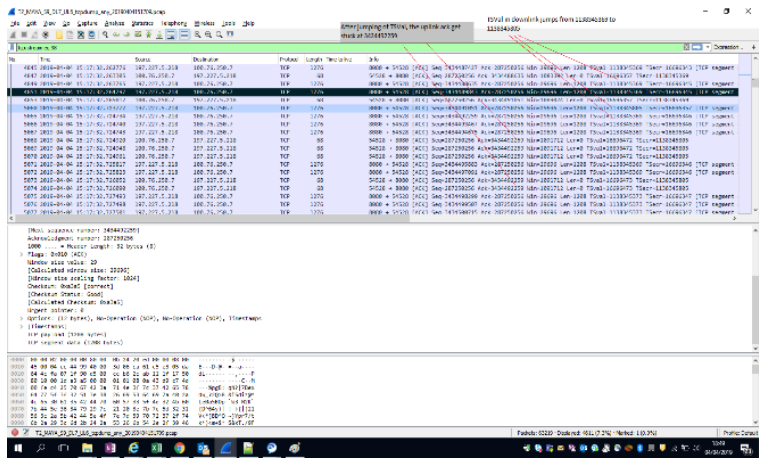

Figure 5: TSVal abnormal behavior trace

Explaining the Figure 5 trace log:

- Things are fine upto packet 4851 (DL TCP SN 3434489843, TSVal 1138345369)

- Next DL packet has TCP SN 3434491051 and TSVal jumps up to 1138345805

- Next packet has TCP SN 3434492259 but TSVal goes back to 1138345369 which was wrong and points to a potential bug in TCP Proxy

Maximum Transmission Unit (MTU) is configured at Egress in each network element along the IP path, originating from the source which can be UE, router or any server sending a packet. Having uniform value across the network can prevent unnecessary TCP fragmentation and its associated packet loss.

Figure 6 depicts the MTU message structure and headers for reference. In general, fragmentation occurs between SGW and PGW.

Figure 6 depicts the MTU message structure and headers for reference. In general, fragmentation occurs between SGW and PGW.

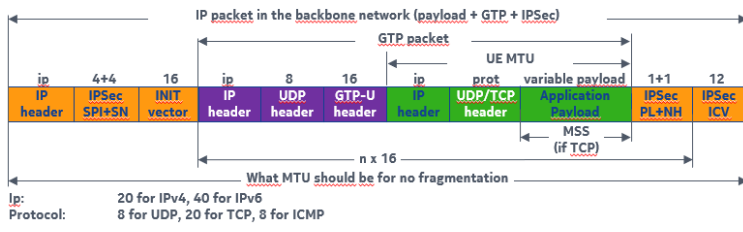

Figure 6: MTU and MSS 3GPP TS 23.060 considerations

\section{a.1 RCA Analysis}

- TCP Proxy function (handled either in GiLAN or within PGW or performed as separate function), if not ordering the packets properly there will be too many Out of Order (OOO) packets error in the network. KPI related to $\mathrm{OOO}$ must be monitored.

- MTU value configured in each network (E2E flow depicted in Figure 7) should be uniform. Note, MTU value will be set different in each market. Inconsistent and different values for each node configured along the path will lead to fragmentation issue that can result in poor performance and packet loss

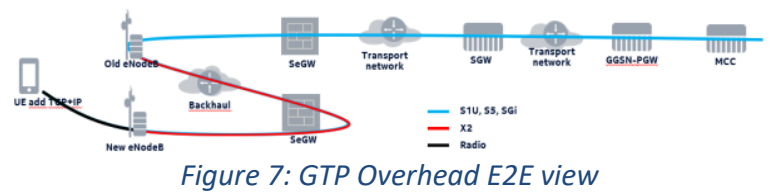

\section{a.2 Recommendations}

- $\quad 3 \mathrm{GPP}$ recommends not to set the MTU value too small to avoid unwanted fragmentation

- MTU minimum value to be at least 1280

- Ensure MTU is consistently configured across nodes

- Monitor OOO related KPI to evaluate packet loss

\section{b. GiLAN DPI configuration issue}

GiLAN solution performs some of the important functions like Deep Packet Inspection (DPI), CGNAT, content filtering, packet filtering and the like.

It will be deployed between PGW and internet, can be deployed either independently or as multiple solutions. The configuration and throttling related policies are required to be managed properly. QoS flow policy defined in GiLAN will have throttling, UL/DL, QoS, bandwidth per user group. 
Recommendation is to perform Configuration Audit in the live network comparing the configured values with the recommended values set by the customer.

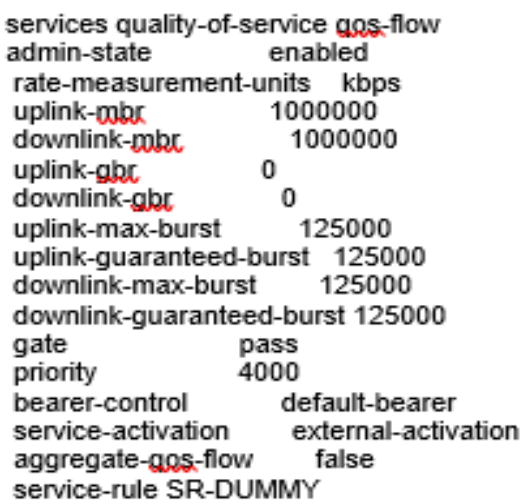

Figure 8: GiLAN Sample QoS Flow settings

Figure 8 depicts the sample config values that will influence the data speed in live network. The values shown are for understanding purpose only and varies operator to operator.

\section{c. Differential and OTT specific issues}

This scenario will be applicable when data services are functional and slow browsing speed is observed for a specific OTT application.

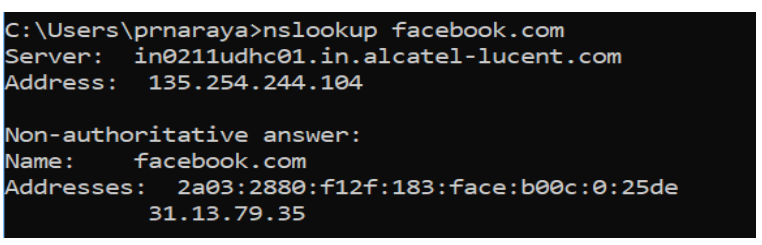

Figure 9: nslookup output

- Check if DNS lookup time is normal for that specific OTT application. Figure 9 provides sample snapshot. Verify if the response time has any induced delay.

- Check if the OTT server is responding to varying byte sizes as depicted in Figure 10.

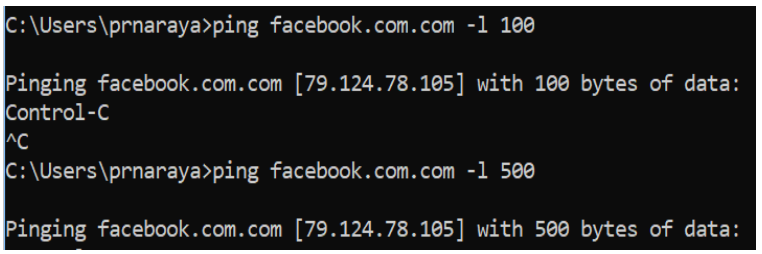

Figure 10: Ping with varying byte sizes

- Verify if the OTT application is allowed in DPI platform. There can be a possibility where the rule is not set, or this application is configured at the bottom of the rule or incorrectly configured.
- For OTT application specific congestion, there can be a possibility where QCI or/and QoS set to lower priority. In such scenario, raise this to customer and agree.

- Traceroute towards the application server, check for delays and path taken. If there is any deviation, detailed investigation is required.

- Issues related to application related browsing or specific source IP pool using different paths for uplink and downlink, it is recommended to route traffic on a common working path. Pre checks on Bandwidth utilization path to be verified before traffic routing.

- Data services affecting a user group, for specific time of the day or day of the week or bundled services then following to be verified:

$$
\begin{array}{ll}
\circ & \text { HSS profile config } \\
\circ & \text { PCRF profile for the user group } \\
\circ & \text { DPI rules }
\end{array}
$$

\section{d. Transmission backbone congestion (SDN)}

In case of Software Defined Networks (SDN) based overlay networks, below listed items impact data browsing experience of the UEs connected to those respective vports.

- $\quad$ Frame Check Sequence (FCS) and Alignment issues on the port

- Traffic will be blackholed if connectivity to Path Computation Element (PCE) was lost

- Connectivity to internet PE's will be lost when default routes in the VRS table was either missing or not followed by the packets.

- BFD link failure or BGP recovery failure. This will result in default routes to internet not advertised properly to all the network nodes.

- Internet traffic not using the shortest-path route to ISP's. This requires detailed investigation.

- To confirm the working of payload traffic specifically on EPC / GiLAN VNF's which are configured with SRIOV networks (catering to high bandwidth need). Verify there is no packet loss observed in these networks using corresponding loop back address.

\section{e. Slow data browsing experience for a specific subscriber}

If data browsing speed issue is reported for a specific subscriber, ensure the impact is for a single user and not to a user group (as per subscription plan, location 
area etc). Following checks requires to be done to identify the root cause:

\section{retLteQO+ServName: RMNIMSQOSO001 pdnMaxReqBwDL : 4294967295 \\ pdnMaxReqBwUL: 4294967295 \\ objectclass: PDNCONTEXT \\ pdncontextId: 2}

Figure 11: QoS Settings

- Check the QoS configured for the user in HSS/ HLR profile, refer Figure 11

- Check the RAT type supported for the UE in the HSS/HLR profile

- Verify whether the Data pack has been exhausted in the PCRF profile. This can result in UE being throttled down (reduced speed) for further data usage.

- If the subscriber is a prepaid user, check if the balance has been exhausted.

- Change the user device and move the SIM card to the new handset. If data works fine on the new handset, UE may be faulty. If problem persists with the changed handset, perform SIM swap.

\section{Tactical solution for faster service restoration}

Service restoration will be the most important action to be done before analyzing the RCA.

- Any network related alarm denotes a straightforward scenario. Identify the corresponding network element and move the traffic to the standby system (Traffic failover).

- Any congestion related alarm denotes a straightforward scenario, move the traffic to the alternate path. This is common to application as well as IP backbone networks.

- Local Preference (LP) values are to be aligned. This is to be checked in scenario's where anycast mechanism was deployed.

- Ensure load balancing is working correctly and as per configuration.

- Check for any inter-site level VIP failures. If yes, switch traffic to standby VIP.

\section{CONCLUSION}

Operational hygiene is key to avoid running into unwanted issues faced in the network. To address concerns over slow data browsing, below listed precautions are to be followed.

- Check recent critical activities performed in network should have speed test/ latency check, part of post validation.
- Request for source and destination IP (in some cases it can be shared)

- Activities that could impact data browsing experience:

- For non-cloud-based network, addition of new User / control IP blades at RNC, PGW etc.

- New IP subnet addition in network for User / Control Plane

- UE pool expansion at PGW

- Access Control List (ACL) configuration in PGW should be in line with the Gi interface's ACL inbound outbound configuration.

○ Node software upgrades

- Critical alarms reported from the overlay network to be monitored

- Stability of the VNFs/PNFs needs to be checked

- If the issue is pertaining to a specific location, time of the day, investigate the radio network, the details are not considered in the scope of this document.

- Awareness of activities happening in the network that might trigger slow data browsing problems, so the necessary pre- and post-checks can be performed.

\section{REFERENCES}

[1] https://www.lightreading.com/ - Topics related to Data traffic issue

[2]3GPP TS 23.060 (etsi.org)

[3] The Evolved Packet Core (3gpp.org) 